\title{
Characterization and isolation of DNA microsatellite primers for Arapaima gigas, an economically important but severely over-exploited fish species of the Amazon basin
}

\author{
IZENI P. FARIAS, ${ }^{*}+$ TOMAS HRBEK, †§ HENNER BRINKMANN,†IRACILDA SAMPAIO $\ddagger$ and \\ AXEL MEYER† \\ *Universidade do Amazonas, Departamento de Biologia, ICB, Manaus, Amazonas, Brazil, +University of Konstanz, Faculty of Biology, \\ Konstanz, Germany, łUniversidade Federal do Pará, Campus de Bragança, Pará, Brazil, §Washington University School of Medicine, \\ Department of Anatomy and Neurobiology, St. Louis, MO, USA
}

\begin{abstract}
Arapaima gigas is a CITES II listed species, but it is also one of the most important food fish of the Amazon basin with a historically very large commercial and subsistence fishery. By the 1980s it became commercially extinct in many areas, and in early 2001 the Brazilian government banned all fishing, although this has not prevented illegal fishing. Therefore we developed 14 variable microsatellite markers for A. gigas to gain a knowledge of its population structure, which is needed to implement a sound management policy, and also to provide us with the forensic tools to manage and monitor this over-exploited fish.
\end{abstract}

Keywords: Amazon, Arapaima gigas, conservation genetics, cross-species amplification, microsatellites, pirarucu

Received 18 September 2002; revision accepted 20 November 2002

Arapaima gigas is one of the most important food fish species of the Amazon basin. Traditionally, Arapaima has formed a significant portion of the diet of Amerindians living near larger bodies of water. Starting as early as the late 18th century, a commercial fishery was developed for Arapaima to satisfy demand for bacalhau, a salted and dried cod for which Arapaima was found to be an excellent freshwater substitute. Its prominence and commercial importance increased during the colonial era as a cheap protein source and its popularity continues to this day. Until the early 1960s, Arapaima appears to have been very abundant near the main Amazonian cities, such as Manaus in the State of Amazonas, Brazil, and Santarém and Belém in the State of Pará, Brazil, which served as centres of export and preparation of bacalhau (Crossa \& Petrere 1999). Due to its enormous commercial importance, Arapaima became increasingly scarce in the 1970s until in the 1980s, it became commercially extinct near larger Amazonian cities (Goulding 1980). In 1975, Arapaima became one of the few fish species listed

Correspondence: Izeni P. Farias, Universidade do Amazonas, Departamento de Biologia, ICB, Manaus, Amazonas, Brazil. Fax: +55 (92) 631-8313, E-mail: izeni@argo.com.br by the Convention on International Trade in Endangered Species (CITES) II convention. In early 2001, the Brazilian government banned all fishing for Arapaima, although this does not prevent illegal fishing, and sale in markets and restaurants. Knowledge of the population structure of A. gigas is needed to implement sound management policies and to provide us with forensic tools to manage and monitor this over-exploited fish. Therefore we developed and report 14 highly variable microsatellite markers for $A$. gigas.

Total genomic DNA was extracted from muscle or liver tissue preserved in $95 \%$ ethanol. The tissue was dissolved and digested with a proteinase $\mathrm{K} /$ sodium dodecyl sulphate (SDS) solution followed by phenol and chloroform extraction, then, addition of $5 \mathrm{M} \mathrm{NaCl}$ followed by $70 \%$ ethanol precipitation of DNA product. Microsatellite loci were isolated and identified from a partial genomic library enriched for CA and CT repeats following the protocol of Tenzer et al. (1999) modified by Garner et al. (2000). Enriched DNA was ligated into an Invitrogen pCR®4 TOPO cloning vector and transformed into chemicallycompetent Escherichia coli cells supplied in the TOPO TA Cloning ${ }^{\circledR}$ kit, following the manufacturer's recommendations. After recovery, the transformed cells were grown 
Table 1 Characteristics of 14 microsatellite loci of 15 Arapaima gigas individuals trom Santarém, Pará, Brazil

\begin{tabular}{|c|c|c|c|c|c|c|c|}
\hline $\begin{array}{l}\text { Locus GenBank } \\
\text { No. }\end{array}$ & $\begin{array}{l}\text { Repeat } \\
\text { motif }\end{array}$ & Primer $\left(5^{\prime}-3^{\prime}\right)$ & $\begin{array}{l}\text { Size range } \\
(\mathrm{bp})\end{array}$ & $\begin{array}{l}\text { No. of } \\
\text { alleles }\end{array}$ & $\begin{array}{l}T_{\mathrm{a}} \\
\left({ }^{\circ} \mathrm{C}\right)\end{array}$ & $H_{\mathrm{O}}$ & $H_{\mathrm{E}}$ \\
\hline $\begin{array}{l}\mathrm{AgCTm} 1 \\
\mathrm{AY} 176172\end{array}$ & $(\mathrm{GT})_{3}(\mathrm{CT})_{12}$ & $\begin{array}{l}\text { F: CCTGTTGCTCATCTGTTATC } \\
\text { R: ATTGCCTCCTGTTCTTGTCG }\end{array}$ & $302-304$ & 2 & 55 & 0.40 & 0.46 \\
\hline $\begin{array}{l}\mathrm{AgCTm} 3 \\
\mathrm{AY} 176173\end{array}$ & $(\mathrm{CT})_{15}$ & $\begin{array}{l}\text { F: ATCTGTTTGTGGGTCTCGAC } \\
\text { R: TGTGAGGACAAGCTCCAGAG }\end{array}$ & 293-299 & 3 & 55 & 0.73 & 0.63 \\
\hline $\begin{array}{l}\mathrm{AgCTm} 4 \\
\mathrm{AY} 176174\end{array}$ & $(\mathrm{CT})_{22}$ & $\begin{array}{l}\text { F: TTTCCCGGACGAGAGAACTG } \\
\text { R: TGTACCAAAGTGATGGAGAG }\end{array}$ & $275-283$ & 3 & 58 & 0.73 & 0.66 \\
\hline $\begin{array}{l}\text { AgCTm5 } \\
\text { AY176175 }\end{array}$ & $(\mathrm{CT})_{29}$ & $\begin{array}{l}\text { F: GCAGCCCCAGTCTTGGAAGG } \\
\text { R: AGCACGGTGAATATCTGTGC }\end{array}$ & $258-280$ & 8 & 58 & 0.67 & 0.87 \\
\hline $\begin{array}{l}\mathrm{AgCTm} 7 \\
\text { AY176176 }\end{array}$ & $(\mathrm{CT})_{29}$ & $\begin{array}{l}\text { F: CCTCTACTTCCTCAACCAGC } \\
\text { R: CAGCACGGTGAATATCTGTG }\end{array}$ & $277-299$ & 6 & 58 & 0.67 & 0.71 \\
\hline $\begin{array}{l}\text { AgCTm8 } \\
\text { AY176177 }\end{array}$ & $\begin{array}{l}(\mathrm{CT})_{5} \mathrm{AT}(\mathrm{CT})_{5} \mathrm{AT}(\mathrm{CT})_{4} \\
\mathrm{TA}(\mathrm{CA})_{13}(\mathrm{CT})_{13}\end{array}$ & $\begin{array}{l}\text { F: TGGAACTCCATTGTGACAGC } \\
\text { R: GACATCTTTTCCAGCTAGCC }\end{array}$ & $272-274$ & 2 & 58 & 0.53 & 0.42 \\
\hline $\begin{array}{l}\mathrm{AgCAm} 2 \\
\mathrm{AY} 176178\end{array}$ & $(\mathrm{CA})_{22}$ & $\begin{array}{l}\text { F: AGCTCTCAGTACTGATGCTG } \\
\text { R: CCGATCATCTGTTTGCTCTG }\end{array}$ & $295-323$ & 7 & 58 & 0.60 & 0.85 \\
\hline $\begin{array}{l}\operatorname{AgCAm} 4 \\
\text { AY176179 }\end{array}$ & $(\mathrm{CA})_{7}$ & $\begin{array}{l}\text { F: CGGGGCTTTTGACTGCAAGC } \\
\text { R: GGAAAGGGGTCGGCTCAGTG }\end{array}$ & $210-212$ & 2 & 55 & $0.00^{*}$ & 0.30 \\
\hline $\begin{array}{l}\text { AgCAm13 } \\
\text { AY176180 }\end{array}$ & $(\mathrm{GTA})_{2}(\mathrm{CA})_{27}$ & $\begin{array}{l}\text { F: TGAAAATCCTGTGGGACCTG } \\
\text { R: CCTAAAAACACATCACACTG }\end{array}$ & $300-334$ & 6 & 58 & 0.73 & 0.80 \\
\hline $\begin{array}{l}\operatorname{AgCAm} 15 \\
\text { AY176181 }\end{array}$ & $(\mathrm{CA})_{19}$ & $\begin{array}{l}\text { F: GGCCTACATCAAGCACTTAA } \\
\text { R: TTACTGGGTTGAGTTTTGAC }\end{array}$ & $226-244$ & 5 & 58 & 0.67 & 0.61 \\
\hline $\begin{array}{l}\text { AgCAm16 } \\
\text { AY176182 }\end{array}$ & $(\mathrm{CA})_{19}$ & $\begin{array}{l}\text { F: CTCCTGGGCATCATGGGTAG } \\
\text { R: TCTGTGTCTCCAGGCAACAG }\end{array}$ & $247-273$ & 6 & 58 & 0.40 & 0.50 \\
\hline $\begin{array}{l}\mathrm{AgCAm} 18 \\
\mathrm{AY} 176183\end{array}$ & $(\mathrm{GA})_{2}(\mathrm{CA})_{9} \operatorname{CTCACT}(\mathrm{CA})_{20}$ & $\begin{array}{l}\text { F: TTACTGAGGGCAATGACACG } \\
\text { R: TGATTACTCAGCAGGTCCTG }\end{array}$ & $357-375$ & 4 & 58 & 0.53 & 0.64 \\
\hline $\begin{array}{l}\operatorname{AgCAm} 20 \\
\text { AY176184 }\end{array}$ & $(\mathrm{CA})_{12}$ & $\begin{array}{l}\text { F: GGAATGAGCAGGTTTCCCAG } \\
\text { R: CTCCCTCTTCTGACATGACG }\end{array}$ & $263-267$ & 3 & 58 & 0.80 & 0.60 \\
\hline $\begin{array}{l}\mathrm{AgCAm} 26 \\
\text { AY176185 }\end{array}$ & $(\mathrm{CA})_{14}$ & $\begin{array}{l}\text { F: ACAGGGACCAGTAAGTGGCC } \\
\text { R: TGCCATAAGCACCGGGTAGG }\end{array}$ & $213-217$ & 3 & 58 & 0.53 & 0.66 \\
\hline
\end{tabular}

All forward primers were labelled with FAM-6, HEX or TET dye. Repeat motif is derived from a sequenced clone; $T_{a^{\prime}}$ locus specific annealing temperature; $H_{\mathrm{O}}$, observed heterozygosity; $H_{\mathrm{E}}$, expected heterozygosity; ${ }^{*}$ significant heterozygote deficiency $(P<0.05)$.

overnight on a $1 \times$ Luria-Bertani (LB) /Amp (Maniatis et al. 1982) agar plate. Individual colonies were picked and re-grown for $6 \mathrm{~h}$ in a 96-well culture plate containing $180 \mu \mathrm{L}$ of liquid $1 \times \mathrm{LB} / \mathrm{Amp}$ solution. Polymerase chain reaction (PCR) amplification using M13 forward (-20) and M13 reverse primers was performed directly on the bacterial cultures following the PCR protocol of Invitrogen. PCR products were purified using Quiagen spin-columns, and sequencing reactions were performed with internally-placed T7 and T3 primers using Perkin Elmer's recommended protocol for BigDye ${ }^{\circledR}$ V2.0 sequencing chemistry. Products were visualized on a Perkin Elmer ABI 3100 Genetic Analyser.

High quality microsatellite repeats were found in 45 of 96 clones sequenced. Primers annealing to flanking regions were designed for the 45 positive clones. PCR was performed in $10 \mu \mathrm{L}$ reaction volumes containing $5.5 \mu \mathrm{L}$ double-distilled $\mathrm{H}_{2} \mathrm{O}, 1.0 \mu \mathrm{L} 10 \times$ buffer $(100 \mathrm{~mm}$ Tris- $\mathrm{HCl}$, $\left.500 \mathrm{~mm} \mathrm{KCl}, 15 \mathrm{~mm} \mathrm{MgCl})_{2}\right), 2.0 \mu \mathrm{L}$ primer mix $(0.2 \mu \mathrm{M}$ each forward and reverse primer), $0.8 \mu \mathrm{L}$ dNTP mix (200 $\mu \mathrm{M}$ each dNTP), 0.2 U Sigma RedTaq ${ }^{\circledR}$ DNA Polymerase, and about 5 ng DNA. PCRs were run on a Perkin Elmer
GeneAmp PCR 9700. An initial denaturation step $\left(94^{\circ} \mathrm{C}\right.$, $2 \mathrm{~min}$ ) was followed by 35 cycles of $10 \mathrm{~s}$ at $94^{\circ} \mathrm{C}, 10 \mathrm{~s}$ at the locus specific annealing temperature (Table 1 ), 30 s at $72{ }^{\circ} \mathrm{C}$, and a final extension step for $60 \mathrm{~min}$ at $72^{\circ} \mathrm{C}$. Sixteen of the primers consistently produced strong single bands and therefore, we labelled the forward primers with the dyes FAM-6, HEX and TET to allow multiplexing. PCR products generated with labelled primers were visualized on a Perkin Elmer ABI 3100 Genetic Analyser. Allele sizes were scored against an internal GeneScan-500 (Rox) size standard. Individuals were genotyped using GENESCAN Analysis 3.7 and GENOTYPER 3.7 software from Perkin Elmer.

Of the 16 loci analysed, two were monomorphic and 14 were variable (Table 1). Levels of variability detected at these 14 loci were high, with the number of alleles ranging from two to seven. Eleven of the 14 microsatellites had observed heterozygosities greater than $50 \%$ (Table 2). Using Arlequin (Schneider et al. 2000) we found a significant heterozygote deficiency $(P<0.05)$ for the locus AgCAm4.

Although $A$. gigas has no close relatives, we tested for cross-species amplification of primers' in Heterotis niloticus, 
Table 2 Cross-species amplitication with Arapaima gigas microsatellite primers

\begin{tabular}{|c|c|c|c|c|c|c|c|c|c|c|c|c|c|c|}
\hline \multirow[b]{2}{*}{ Species } & \multicolumn{14}{|l|}{ Locus } \\
\hline & $\begin{array}{l}\mathrm{AgCT} \\
\mathrm{m} 1\end{array}$ & $\begin{array}{l}\mathrm{AgCT} \\
\mathrm{m} 3\end{array}$ & $\begin{array}{l}\mathrm{AgCT} \\
\mathrm{m} 4\end{array}$ & $\begin{array}{l}\mathrm{AgCT} \\
\mathrm{m} 5\end{array}$ & $\begin{array}{l}\mathrm{AgCT} \\
\mathrm{m} 7\end{array}$ & $\begin{array}{l}\mathrm{AgCT} \\
\mathrm{m} 8\end{array}$ & $\begin{array}{l}\mathrm{AgCA} \\
\mathrm{m} 2\end{array}$ & $\begin{array}{l}\mathrm{AgCA} \\
\mathrm{m} 4\end{array}$ & $\begin{array}{l}\mathrm{AgCA} \\
\mathrm{m} 13\end{array}$ & $\begin{array}{l}\mathrm{AgCA} \\
\mathrm{m} 15\end{array}$ & $\begin{array}{l}\mathrm{AgCA} \\
\mathrm{m} 16\end{array}$ & $\begin{array}{l}\mathrm{AgCA} \\
\mathrm{m} 18\end{array}$ & $\begin{array}{l}\mathrm{AgCA} \\
\mathrm{m} 20\end{array}$ & $\begin{array}{l}\mathrm{AgCA} \\
\mathrm{m} 26\end{array}$ \\
\hline Gnathonemus petersii & ++ & ++ & ++ & - & ++ & + & ++ & - & ++ & - & ++ & - & ++ & - \\
\hline Heterotis niloticus & - & - & - & - & - & - & + & - & - & - & + & - & ++ & - \\
\hline Osteoglossum bicirrhosum & - & ++ & - & - & ++ & ++ & - & - & - & - & - & + & ++ & - \\
\hline Osteoglossum ferreirai & - & - & - & - & + & - & - & - & - & - & - & - & - & - \\
\hline Pantodon buchholzi & ++ & ++ & - & - & ++ & + & + & - & ++ & - & - & ++ & - & - \\
\hline
\end{tabular}

++ indicates a very good, and potentially useful amplification; + indicates weaker amplification or multiple bands;

- indicates no amplification. Amplifications were done at $50{ }^{\circ} \mathrm{C}$ annealing temperature, the rest of the protocol was same as for A. gigas.

the African sister species of $A$. gigas, as well as more distantly related osteoglossoform fish including Osteoglossum bicirrhosum and O. ferreirai, both from the Amazon basin, Pantadon buchholzi from West Africa and the mormyrid Gnathonemus petersi from Central Africa (Table 2). All reactions were carried out at a $50^{\circ} \mathrm{C}$ annealing temperature, with the rest of the amplification protocol the same as that for A. gigas. Loci were judged as potentially useful in other species if they amplified as a strong distinct band in the size range near that of $A$. gigas; weaker bands or multiple bands were scored as questionable but of potential use (Table 2). PCR fragments for other species were not sequenced to determine homology or the presence of a repeat element.

\section{Acknowledgements}

The authors thank Barbara Gautschi and Trent Garner (ETH Zürich) for technical assistance and Marcelo Crossa (IPAM Santarém) for collecting the Arapaima gigas samples. Sébastien Lavoue provided samples of Heterotis niloticus. This research was supported by Universidade do Amazonas (to I.P.F.), the National Science Foundation (INT-0002213 to T.H.), EMBRAPA
(PRODETAB)/CNPq and Universidade Federal do Pará (to I.S.), and the University of Konstanz and the Deutsche Forschungsgemeinschaft (to A.M.). Permission to collect tissue samples has been granted by IBAMA.

\section{References}

Crossa M, Petrere M Jr (1999) Morphometric relationships and indirect determination of the length frequency structure of the pirarucu Arapaima gigas Cuvier. Fisheries Management and Ecology, 6, 173-186.

Garner TWJ, Gautschi B, Röthlisberger S, Reyer H-U (2000) A set of CA repeat microsatellite markers derived from the pool frog, Rana lessonae. Molecular Ecology, 9, 2173-2175.

Goulding M (1980) Fishes and the Forest. University of California Press, Los Angeles.

Maniatis T, Fritsch EF, Sambrook J (1982) Molecular Cloning: a Laboratory Manual. Cold Spring Harbor Laboratory Press, Cold Spring Harbor, NY.

Schneider S, Roessli D, Excoffier L (2000) Arlequin ver 2000: A software for population genetic data analysis. University of Geneva, Genetics and Biometry Laboratory.

Tenzer I, Sd I, Morgante M, Gessler C (1999) Identification of microsatellite markers and their application to population genetics of Venturia inaequalis. Phytopathology, 89, 748-753. 